\title{
Editorial
}

\section{Do we all need to have TEE capability?}

Transoesophageal echocardiography (TEE) has been described as "the safest, most reliable, most rapid, and most cost-effective tẹnnique for the assessment of many life-threatening "cardiovascular problems." It is used routinely in $35 \%$ of cardiac surgical centres in the United States. This issue of the Journal contains articles by Beique et $a l^{3}$ and Oxorn et al. ${ }^{2}$ on the use of TEE. At this juncture, it may be timely to examine certain issues surrounding the application of this technology to anaesthesia practice in Canada and to ask whether we all need to become proficient in transoesophageal echocardiography. To formulate an answer, one approach might be to examine the data available for some of the suggested applications of this technology to anaesthesia, ${ }^{4,5}$ and critical care, ${ }^{6}$ and to reflect on certain practical issues related to its introduction into Canadian anaesthetic practice.

\begin{abstract}
Assessment of stroke volume and cardiac output
Measurement of stroke volume is best accomplished during TEE by edge detection of the endocardial border. ${ }^{7,8}$ Even in skilled hands, in up to $24 \%$ of patients, technical difficulties will preclude visualization of the entire circumference of the heart (and thus prevent calculation of the endocardial border derived stroke volume (fractional area change)). In addition, the development of wall motion abnormalities will cause further error in the measurement. ${ }^{7,8}$

While there is good correlation with measurement of left ventricular volume as assessed by angiography, and while useful as a prognostic indicator, the value of online measurement of ejection fraction by $\mathrm{TEE}^{9}$ has not been clearly defined. It is acknowledged that TEE measures of left ventricular volume status may represent a better "gold standard" for intraoperative monitoring of cardiac filling volume and that there is poor correlation between TEE measures of end-diastolic volume and more traditional surrogate measures such as pulmonary artery occlusion pressure. However, the real question is
\end{abstract}

From the Department of Anaesthesia, Dalhousie University, Halifax, Nova Scotia, Canada. does the use of TEE detect deteriorating cardiac function so much earlier than more traditional measures such as changes in pulmonary artery occlusion pressure, blood pressure, ECG, or cardiac output that its use improves patient outcome? ${ }^{10}$ This would be a compelling argument for more routine use of such a monitoring device but data are unavailable. Finally, it must be pointed out that simultaneously measured thermodilution and TEE cardiac output may vary by up to $30 \% .^{11}$ Which of the two measurement techniques reflects the "true" situation and under what circumstances false positive or negative results are obtained has not been adequately studied.

\section{Monitor of myocardial ischaemia}

Changes in segmental wall motion, as detected by TEE, are felt to be indicative of myocardial ischaemia and may be more sensitive than the ECG. ${ }^{12}$ However, as a monitor of the development of ischaemia there are limitations to the use of TEE. ${ }^{13}$ Severe hypokinesis, the most frequent TEE finding suggestive of ischaemia, has only limited sensitivity and specificity. ${ }^{14}$ Just as ischaemia detected by the ECG is subject to the number of leads monitored and their location, detection of ischaemia by TEE is limited by the view used to detect the development of wall motion abnormalities, ${ }^{15}$ and may be operator dependent. ${ }^{16}$ Absence of segmental wall motion abnormalities (SWMA) does not mean absence of ischaemia ${ }^{13}$ and persistent SWMA do not always lead to myocardial infarction. ${ }^{14}$ There is poor concordance between ischaemia as detected by TEE and ischaemia detected by other measures (e.g., ECG, PAOP). ${ }^{13,14}$. Which method is the most accurate is not well defined. Not every wall motion abnormality can be ascribed to ischaemia. ${ }^{17}$ The definition of what constitutes a clinically important SWMA has only recently been standardized, making comparison of results obtained in early trials difficult to compare with later studies.

The introduction of the TEE probe before induction of anaesthesia and tracheal intubation is unlikely to become common practice. Thus, there are at least two periods when ischaemia detection has traditionally been 
felt to be important but that will be missed, i.e., at induction and at intubation. Without an awake baseline, how do TEE practitioners decide what represents new ischemic changes ? $^{14}$ Given this limitation and its cost, it is unlikely that TEE will replace the ECG as a commonly used cardiac monitor.

\section{Abnormal cardiac anatomy of blood flow}

It is in the assessment of altered cardiac anatomy e.g., valve repair and function that TEE has an unquestionably important role to play. ${ }^{18}$ It has been used to advantage for detection, and repair, of valves and congenital lesions, and for detection of the aortic atherosclerosis. ${ }^{19}$ Its intraoperative use in this regard raises some thorny issues. Who is responsible for making the diagnosis anaesthetist, surgeon or cardiologist? ${ }^{10}$ Practice in this regard is quite variable. ${ }^{21}$ What level of training is required to enable the anaesthetist to make the right diagnosis. ${ }^{22}$ What are the medicolegal consequences of missing the diagnosis? As we cannot all go away for six months to learn TEE, ${ }^{22}$ how will department members train each other? Will we further reduce OR availability until we have a cadre of physician echocardiographers capable of providing the service? This could be divisive for departments and could lead to a two tier system those with and those without a TEE "ticket," particularly if TEE is reimbursed in a manner similar to that for insertion of a pulmonary artery catheter. How will quality assurance be implemented and skills maintained ${ }^{23}$ as a certain number of TEE examinations are required to maintain proficiency? Finally, will there be adequate exposure to cases requiring TEE for everyone to retain their skills as echocardiographers or is it preferable to have a limited number of individuals who do sufficient examinations to retain their TEE skills?

\section{Trauma}

The anatomical definition produced by TEE has established a role for TEE in the diagnosis of chest injury following trauma, particularly aortic injury. ${ }^{19,24}$ Experience demonstrates that TEE can often be safely performed faster than angiography ${ }^{24}$ and may avoid some of the complications associated with angiography e.g., renal compromise due to the dye load. Concerns generated by TEE in this setting include its performance in patients without a protected airway, inability to visualize certain portions of the aorta, and availability of trained personnel to perform the examination at all hours of the day or night. Anaesthetists may well have a supportive role to play here. False positive examinations are reported. ${ }^{24}$ While not yet reported, the consequences of making a false negative report in this scenario may be disastrous (40\% mortality within $24 \mathrm{hr}$ ).

\section{Safety}

As with all invasive monitors, things can and do go wrong during the insertion of a TEE probe and subsequent monitoring. ${ }^{25}$ Some are minor - trauma to lips, etc., while others are potentially life-threatening e.g., pharyngeal rupture. ${ }^{19}$ Death has been reported. ${ }^{25}$ One other issue needs consideration - this monitor is quite capable of mesmerizing the operator. So many knobs, pictures, etc., may make it difficult to concentrate on the patient and task at hand.

\section{Cost}

The capital purchase costs for the equipment are substantial. Maintenance and repair costs must also be factored into the decision to purchase a TEE. It is one thing to drop a $\$ 60.00$ catheter on the floor - quite another to drop a probe worth thousands of dollars. These considerations will limit many institutions in Canada, given the current fiscal climate.

\section{Practical issues}

Insertion of a TEE takes time and the equipment is bulky. Where anaesthetic practices are primarily rapid turnover cases, the use of TEE will have limited applicability, except in very high risk cases. In addition, certain surgical positions, e.g., prone, may further limit its use in non-cardiac procedures.

\section{Conclusions}

Given these considerations, and in the absence of data to the contrary, the answer to the question of whether we all need to be proficient in TEE must, in my opinion, at the present time, be no. It will have a role to play in centres where cardiac surgery or treatment of major trauma are performed. Apart from these areas, the onus is on TEE investigators, using appropriately rigorous investigative methods, to demonstrate why the rest of us should develop TEE skills. This is not to deny that the role for TEE may not expand but, before more widespread application of the technology (and its cost) can be justified, further study is required.

\section{Devons-nous tous être compétents en ETO?}

On a décrit l'échographie transoesophagienne (ETO) comme « la technique proportionnellement à son coût la 
plus sûre, la plus fiablę et la plus efficace pour diagnostiquer plusieurs conditions cardiaques menaçantes pour la vie ". ${ }^{1}$ Elle est utilisée dans $35 \%$ des centres de chirurgie cardiaque aux Etats-Unis. Ce numéro du Journal contient des articles écrits par Béique $e t a l^{3}$ et Oxorn et al. ${ }^{2}$ sur l'utilisation de l'ETO. Le temps est donc venu de jeter un coup d'oeil sur certains des aspects propres à cette technique appliquée à l'exercice de l'anesthésie au Canada et de se demander s'il est important que nous devenions tous compétents en échographie transoesophagienne. Pour pouvoir répondre à cette question, il est bon d'examiner les applications de cette technologie en anesthésie ${ }^{4,5}$ et en réanimation ${ }^{6}$ et de réfléchir sur les aspects pratiques de son introduction au Canada.

\section{Mesure du volume d'éjection et du débit cardiaque}

Le meilleur moyen de mesurer le débit cardiaque par ETO consiste à déterminer les limites de l'endocarde., ${ }^{7,8}$ Même entre les mains 'd'experts, et dans jusqu'à $24 \%$ des cas, les difficultés techniques empêchent de visualiser la surface cardiaque dans sa totalité, prévenant ainsi le calcul du volume d'éjection par le changement de la surface endocardique. En outre, l'apparition d'altérations de la contractilité localisées augmentera les causes d'erreurs. ${ }^{7.8}$

Alors que la corrélation avec la mesure angiographique du volume ventriculaire gauche est bonne, et bien qu'elle soit utile comme indicateur de pronostic, la valeur de la mesure de la fraction d'éjection «en ligne » par ETO $^{9}$ n'est pas clairement établie. On reconnaît qu'à l'ETO, la mesure du volume ventriculaire gauche peut représenter une meilleure norme de monitorage peropératoire du volume de remplissage cardiaque; la corrélation est pauvre entre les mesures du volume télédiastolique par ETO et les mesures de substitut traditionnelles comme les changements de pression pulmonaire capillaire bloquee (PPCB). Cependant, une question doit être posée: est-ce que l'ETO signale une détérioration de la fonction cardiaque de façon tellement plus précoce que les méthodes traditionnelles comme les changements de la PPCB, de la pression artérielle, de l'ECG, ou du débit cardiaque au point où elle influence le devenir du patient? ${ }^{10}$ Ceci représenterait un argument concluant pour l'usage courant de l'ETO comme moniteur mais nous ne possédons pas de données suffisantes pour le prouver. Finalement, il faut mentionner que la mesure simultanée du débit cardiaque par thermodilution et par ETO peut varier de 30\%." Des deux mesures, celle qui reflète le mieux la réalité et les circonstances qui provoquent les faux positifs et les faux négatifs n'ont pas encore été suffisamment étudiées.

\section{Moniteur d'ischémie myocardique}

Les changements de la fonction segmentaire révélés par ETO sembleraient indiquer l'ischémie myocardique tout en étant plus sensibles que l'ECG. ${ }^{12}$ Cependant, l'ETO a ses limites comme moniteur d'ischémie myocardique. ${ }^{13}$ A l'ETO, l'hypokinésie, signe le plus constant de l'ischémie, n'a qu'une sensibilité et une spécificité limitées. ${ }^{14} \mathrm{De}$ la même façon quel'ischémie qui, lorsque détectée par l'ECG, est tributaire du nombre de dérivations et leurs sites, la détection de l'ischémie par l'ETO est limitée par l'imagerie utilisée pour détecter le développment des anomalies de la fonction segmentaire ${ }^{15}$ et est dépendante du manipulateur. ${ }^{16}$ L'absence d'anomalies de la fonction segmentaire ne signifie pas absence d'ischémie ${ }^{13}$ et les anomalies persistantes ne conduisent pas nécessairement à l'infarctus du myocarde. ${ }^{14} \mathrm{La}$ concordance est faible entre l'ischémie détectée par ETO et par d'autres mesures (par ex., ECG, PPCB).$^{13,14}$ La méthode la plus précise n'a pas encore été déterminée. Toutes les altérations de la fonction segmentaire ne peuvent être attribuées à l'ischémie. ${ }^{17} \mathbf{L a}$ standardisation de la définition du dysfonctionnement segmentaire cliniquement significatif étant très récente, la comparaison des résultats obtenus dans le passé avec ceux des études subséquentes est difficile.

Il est peu probable que l'introduction de la sonde d'ETO avant l'induction de l'anesthésie et l'intubation de la trachée devienne une pratique courante. Les deux périodes les plus importantes au cours desquelles la détection de l'ischémie a toujours été traditionnellement considéré comme essentielle, l'induction et l'intubation, sont donc écartées. Sans la ligne de base de l'état vigile, comment les échographistes peuvent-ils identifier les altérations récentes causées par l'ischémie? ${ }^{14}$ Avec ses limites et ses coûts, il est peu probable quel'ETO remplace un jour l'ECG comme moniteur cardiaque de base.

\section{Les anomalies cardiaques anatomiques et circulatoires}

L'ETO joue un rôle évident dans l'évaluation des cardiopathies valvulaires et fonctionnelles. ${ }^{18}$ L'ETO a été utilisée avantageusement pour détecter et réparer les valves et les lésions congénitales cardiaques et pour détecter l'athérosclérose aortique, ${ }^{19}$ mais son utilisation peropératoire soulève des questions complexes. Qui est responsable du diagnostic? L'anesthésiste, le chirurgien ou le cardiologue ${ }^{20}$ Sur ce point, la pratique est très variable. ${ }^{21}$ Quel niveau de formation permet à l'anesthésiste de faire un diagnostic correct? ${ }^{1,22}$ Quelles sont les conséquences médicolégales d'un diagnostic erroné? Comme nous ne pouvons pas tous nous absenter pendant six mois pour apprendre l'ETO, ${ }^{22}$ comment 
les membres d'un département formeront-ils leurs collègues? Les heures de disponibilité en salle d'opération devront-elles diminuer pour permettre la formation d'une équipe d'échographistes suffisante pour fournir des services adéquats? Ceci pourrait diviser les départements en deux catégories: les compétents en ETO et les autres, spécialement si l'ETO est rémunérée de la même façon que l'a été l'insertion des cathéters dans l'artère pulmonaire. Comment alors seront appliqués l'assurance-qualité et le maintien de la compétence ${ }^{23}$ Un certain nombre d'examens à l'ETO est effectivement nécessaire pour le maintien de la compétence. Finalement, il y aura-t-il un nombre suffisant d'ETO pour permettre à chacun de garder sa compétence d'échographiste ou sera-t-il préférable de limiter l'accès à l'ETO à un nombre limité d'individus qui pourront réaliser assez d'ETO pour maintenir cette compétence?

\section{Les traumatismes}

La définition anatomique obtenue par ETO a confirmé, son rôle dans le diagnostic des traumatismes du thorax et plus particulièrement ceux de 1'aorte. ${ }^{19,24}$ L'expérience a démontré que l'ETO peut être réalisée plus rapidement, avec moins de risques que l'angiographie ${ }^{24}$ et peut éviter plusieurs des complications associées, comme l'insuffisance rénale par surcharge de matériel de contraste. Il faut toutefois se préoccuper dans ces circonstances qu'elle sera réalisée sur des patients dont les voies respiratoires sont mal protégées, que certaines portions de l'aorte sont impossible à visualiser, et que le personnel formé pour cet examen n'est pas disponible à toute heure du jour et de la nuit. Les anesthésistes auront un rôle de soutien aussi à jouer. Des faux positifs ont de plus été décrits. ${ }^{24}$ Même si elles n'ont pas encore été rapportées, les conséquences d'une interprétation négative erronée dans un tel scénario peuvent être désastreuses ( $40 \%$ de décès en $24 \mathrm{~h}$ ).

\section{La sécurité}

Comme avec tous les moniteurs effractifs, il arrivent des accidents pendant l'insertion de la sonde d'ETO et le monitorage subséquent. ${ }^{25}$ Quelques-uns sont mineurs, comme les traumatismes labiaux alors que plusieurs sont potentiellement fatals comme la rupture du pharynx. ${ }^{19}$ On a déjà rapporté des décès. ${ }^{25} \mathrm{Il}$ faut aussi considérer d'autres aspects, ce moniteur peut mesmériser son utilisateur. Cet assemblage des boutons, contrôles, images, etc., peut faire oublier le patient et la tâche à accomplir.

\section{Les coûts}

Le capital à investir pour l'achat de l'appareil est con- sidérable. Les coûts d'entretiens et de réparation doivent aussi être considérés si on songe à l'achat d'un ETO. La différence est énorme entre échapper sur le plancher un cathéter de $60,00 \$$ et une sonde de plusieurs milliers de dollars. Dans le climat fiscal actuel, ces considérations auront un impact négatif pour plusieurs institutions au Canada.

\section{Aspects pratiques}

L'insertion d'un ETO prend du temps et l'appareil est encombrant. Là ou la pratique de l'anesthésie est surtout caractérisée par un roulement rapide des cas, l'ETO sera difficilement utilisable excepté lorsque les malades présenteront de très haut risques. En outre, certaines positions comme le décubitus ventral peuvent restreindre son utilisation.

\section{Conclusions}

Avec toutes ce considérations et en absence de données contraires, la réponse à la question que nous avons posée sur la compétence généralisée en ETO, est selon moi, négative. Cependant, l'ETO aura un rôle à jouer là où se pratique le chirurgie cardiaque et celle des grands traumatisés. A l'exception des ces domaines, le fardeau de la preuve est entre les mains des chercheurs qui, avec des méthodes rigoureuses, devront démontrer pourquoi tous devraient développer une compétence en ETO. On ne peut nier que le rôle de l'ETO pourrait prendre de l'expansion, mais avant de justifier l'application générale de cette technologie (et son coût), il faudra l'étudier encore plus.

\section{References}

1 Cahalan $M K$, Foster $E$. Training in transesophageal echocardiography: in the lab or on the job? (Editorial). Anesth Analg 1995; 81: 217-8.

2 Oxorn D, Edelist $G$, Stafford Smith $M$. An introduction to transoesophageal echocardiography: II. Clinical applications. Can J Anaesth 1996; 43: 278-94.

3 Béïque F, Joffe D, Kleiman S. An introduction to transoephageal echocardiography: I. Basic principles. Can J Anaesth 1996; 43: 252-77.

4 Bryan AJ, Barzilai B, Kouchoukos NT. Transesophageal echocardiography and adult cardiac operations. Ann Thorac Surg 1995; 59: 773-9.

5 Dan M, Bonato R, Mazzucco A, et al. Value of transesophageal echocardiography during repair of congenital heart defects. Ann Thorac Surg 1990; 50: 637-43.

6 Foster E, Schiller NB. Transesophageal echocardiography in the critical care patient. Cardiol Clin 1993; 11 : 489-503.

7 Cahalan MK, Ionescu P, Melton HE Jr, Adler S, Kee LL, Schiller NB. Automated real-time analysis of intraopera- 
tive transesophageal echocardiograms. Anesthesiology 1993; 78: 477-85.

8 Pinto FJ, Siegel LC, Chenzbraun A, Schnittger I. On-line estimation of cardiac output with a new autometed border detection system using transesophageal echocardiography: a preliminary comparison with thermodilution. J Cardiothorac Vasc Anesth 1994; 8: 625-30.

9 Smith MD, MacPhail B, Harrison MR, Lenhoff SJ, DeMaria AN. Value and limitations of transesophageal echocardiography in determination of left ventricular volumes and ejection fraction. J Am Coll Cardiol 1992; 19: 1213-22.

10 Watanabe $S$, Buffington $C W$. Speed and sensitivity of mechanical versus electrographic indicators to mild or moderate myocardial ischemia in the pig. Anesthesiology 1994; 80: 582-94.

11 Stoddard MF, Prince CR, Ammash N, Goad JL, Vogel RL. Pulsed Doppler transesophageal echocardiographic determination of cardiac output in human beings: comparison with thermodilution technique. Am Heart J 1993; 126 : 956-62.

12 Smith IS, Cahalan MK, Benefiel DJ, et al. Intraoperative detection of myocardial ischemia in high-risk patients: electrocardiography versus two-dimensional transesophageal echocardiography. Circulation 1985; 72: 1015-21.

13 Eisenberg $M J$, London MJ, Leung JM, et al. Monitoring for myocardial ischemia during noncardiac surgery. A technology assessment of transesophageal echocardiography and 12-lead electrocardiography. JAMA 1992; 268: 210-6.

14 London MJ, Tubau JF, Wong MG, et al. The "natural history" of segmental wall motion abnormalities in patients undergoing noncardiac surgery. Anesthesiology 1990; 73: 644-55.

15 Chung F, Seyone C, Rakowski H. Transesophageal echocardiogram may fail to diagnose peri-operative myocardial infarction. Can J Anaesth 1991; 38: 98-101.

16 Konstadt SN, Abrahams HP, Nejat M, Reich DL. Are wall thickening measurements reproducible? Anesth Analg 1994; 78: 619-23.

17 Pandian NG, Skorton DJ, Collins SM, Falsetti HL, Burke $E R, K e r b e r R E$. Heterogeneity of left ventricular segmental wall thickening and exursion in 2-dimensional echocardiograms of normal human subjects. Am J Cardiol 1983; 51: 1667-73.

$18 D^{\prime} A m b r a M$. Is intraoperative echocardiography a useful monitor in the operating room? Ann Thorac Surg 1993; 56: S83-5.

19 Daniel WG, Mügge A. Transesophageal echocardiography. N Engl J Med 1995; 332: 1268-79.

20 Hodgins L, Kisslo JA, Mark JB. Perioperative transesophageal echocardiography: the anesthesiologist as cardiac diagnostician. Anesth Analg 1995; 80: 4-6.
21 Poterack KA. Who uses transesophageal echocardiography in the operating room? Anesth Analg 1995; 80: 454-8.

22 Savage RM, Licina MG, Koch CG, et al. Educational program for intraoperative transesophageal echocardiography. Anesth Analg 1995; 81: 399-403.

23 Rafferty $T$, LaMantia KR, Davis E, et al. Quality assurance for intraoperative transesophageal echocardiography monitoring: a report of 846 procedures. Anesth Analg 1993; 76: 228-32.

24 Smith MD, Cassidy JM, Souther S, et al. Transesophageal echocardiography in the diagnosis of traumatic rupture of the aorta. N Engl J Med 1995; 332: 356-62.

25 Daniel WG, Erbel $R$, Kasper W, et al. Safety of transesophageal echocardiography. A Multicenter Survey of 10,419 examinations. Circulation 1991; 83: 817-21. 